\title{
Imaging Kinesin-Mediated Transport in Cultured Hippocampal Neurons
}

\author{
Stefanie Kaech, ${ }^{* *}$ Chun-Fang Huang, ${ }^{*}$ Cheng Fang, * Brian Jenkins, ${ }^{*}$ and Gary Banker* \\ *The Jungers Center for Neurosciences Research and **The Advanced Light Microscopy Core, \\ Oregon Health Science University, Portland, OR 97239
}

Dissociated-cell cultures from rat or mouse hippocampus have become a standard model to study many facets of nerve cell biology and development. Because the neurites that form in such cultures are quite thin and grow out directly on the surface of glass coverslips, such cultures are readily amenable to high resolution, live-cell imaging. We are particularly interesting in the mechanism that underlie the differential targeting and transport of membrane proteins to axons or dendrites. We will describe the methods we use for imaging the transport of vesicles that deliver proteins to axons and dendrites and for evaluating the translocation of particular kinesin motor proteins on axonal and dendritic microtubules.

By expressing axonal or dendritic membrane proteins tagged with GFP or its color variants, it is possible to visualize the vesicles that transport these proteins to their appropriate domains. Dendritic and axonal proteins are sorted into different vesicles as they leave the Golgi complex. Axonal carriers are long and tubular whereas dendritic carriers are small and round or only slightly elongated. The two sets of vesicles have distinctly different patterns of transport. Vesicles that carry axonal proteins travel bidirectionally in both axons and dendrites, but their transport is biased toward the axon--about 3 times more carriers enter the axon than enter the average dendrite. Vesicles containing dendritic proteins travel bidirectionally in dendrites, but are virtually excluded from the axon. By expressing different pairs of dendritic proteins labeled with different GFP color variants, we identified at least two different populations of Golgiderived vesicles carrying dendritic proteins. We found only a single class of Golgi-derived carriers that contain axonal plasma membrane proteins.

By imaging the accumulation of truncated, constitutively active kinesins, it is possible to assess the selectivity of kinesin motor domain translocation along neuronal microtubules. Based on this assay, some kinesin motors accumulate exclusively at axonal tips, indicating that they translocate preferentially on axonal microtubules; other kinesins accumulate with equal efficiency at tips of both axons and dendrites. Closely related kinesins from the same subfamily may differ in their translocation preference by this assay.

Early in development, before axon specification, axon-preferring kinesins transiently accumulate in different neurites, which implies that the molecular differences that mark different microtubule populations can be rapidly modulated. Posttranslational modifications of tubulin can regulate the efficiency of kinesin translocation and our evidence indicates manipulations that globally enhance tubulin modifications and eliminate differences in posttranslational modifications between axons and dendrites can alter the translocation preferences of kinesins. These treatments cause kinesins that are normally axon-selective to accumulate in both axons and dendrites, indicating that tubulin posttranslational modifications contribute to the selective translocation of axonal kinesins. 
This research was supported by NIH grant MH MH66179 and a National Multiple Sclerosis Society Center Grant. 